\title{
BMJ Open Discrete choice experiment (DCE) to quantify the influence of trial features on the decision to participate in cystic fibrosis (CF) clinical trials
}

\author{
Rebecca Anne Dobra (D , ${ }^{1,2}$ Marco Boeri, ${ }^{3}$ Stuart Elborn, ${ }^{4}$ Frank Kee, ${ }^{5}$ \\ Susan Madge, ${ }^{6}$ Jane C Davies ${ }^{1,2}$
}

To cite: Dobra RA, Boeri M, Elborn S, et al. Discrete choice experiment (DCE) to quantify the influence of tria features on the decision to participate in cystic fibrosis (CF) clinical trials. BMJ Open 2021;11:e045803. doi:10.1136/ bmjopen-2020-045803

- Prepublication history for this paper is available online. To view these files, please visit the journal online (http://dx.doi org/10.1136/bmjopen-2020045803).

Received 20 0ctober 2020 Revised 21 January 2021 Accepted 04 February 2021

Check for updates

(c) Author(s) (or their employer(s)) 2021. Re-use permitted under CC BY-NC. No commercial re-use. See rights and permissions. Published by BMJ.

For numbered affiliations see end of article.

Correspondence to Dr Rebecca Anne Dobra; r.dobra@imperial.ac.uk

\section{ABSTRACT}

Introduction Engaging people with cystic fibrosis (CF) in clinical trials is critical to improving outcomes for this fatal disease. Following extensive exploration of engagement in CF trials we believe six key concepts require a quantitative understanding of their influence in the current CF trials landscape including how controversial issues like placebos, washouts, stipend provision and location of trial visits are viewed by the CF community and how these might be modified depending on the type of medicine being investigated and the mechanism of access to the drug on trial completion.

Methods and analysis We have designed and will administer an online discrete choice experiment to elicit and quantify preferences of people with CF for these trials' attributes and estimate the relative importance of an attribute when choosing to participate in a trial. The crosssectional data generated will be explored using conditional multinomial logit model. Mixed logit models such as the random-parameters logit and a latent class models will be used to explore preference heterogeneity. To determine the relative importance of an attribute, the difference between the attribute level with the highest preference weight and the level with the lowest preference weight will be calculated.

Ethics and dissemination Imperial College London Joint Research Compliance Office has granted ethical approval for this study. Patient consent will be sought following full explanation. No identifying information will be collected. Dissemination will be via international conferences, peer-review publication and patient accessible forums. Major CF trials networks have agreed to incorporate our findings into their review process, meaning our results can realistically influence and optimise CF trial delivery. PROSPERO registration number CRD42020184886.

\section{INTRODUCTION}

Approximately 11000 people in the UK have cystic fibrosis (CF). ${ }^{1}$ Despite substantial progress, CF still carries a high symptom and treatment burden, and a significantly reduced life-expectancy. ${ }^{1}$ Recent breakthroughs in modulator therapies offer substantial hope for improved health for nearly $90 \%$ of the CF
Strengths and limitations of this study

- Discrete choice experiments (DCEs) bridge the gap between classical experimental methods investigating decision making which are constrained by practical and ethical difficulties, and descriptive methods which introduce numerous sources of bias and necessitate small sample sizes, posing questions about validity and generalisability.

- This DCE was developed following good research practices defined by International Society for Pharmacoeconomics and Outcomes Research guidelines and standard practice in preference studies and has a number of strengths derived from the use of best practices.

- There are some unavoidable potential sources of bias, including the selection bias seen with all online voluntary surveys, information bias from explanation of complex information within the survey, residual confounding bias and the differences between stated and real-world preferences.

- We have made every effort to address potential biases, including careful design of realistic scenarios, extensive pretesting with relevant stakeholders, advertising through multiple channels and analysis using advanced statistical techniques. Where potential bias cannot be mitigated, we acknowledge the source of bias.

Dur collaboration with the major cystic fibrosis (CF) trials networks in Europe and the UK mean our results can realistically influence and optimise CF trial delivery of clinical trials and enhance their acceptability to people with CF in Europe and beyond.

adolescent and adult population. ${ }^{2} 3$ However, while hugely exciting, these breakthroughs do not represent a cure. Much work remains to identify treatments for those with rare mutations, to ensure that treatment starts as early as is safe and practicable, to optimise the choice of modulator therapies and regimen and improve traditional therapies targeting the downstream consequences of established disease. ${ }^{45}$ There are more than 100 drugs in 
the development pipeline, ${ }^{6}$ but without patient participation, clinical trials will not succeed. Therefore, engaging people with $\mathrm{CF}$ in research is essential to improve outcomes for this fatal disease.

Making research more patient-centred is a priority of research organisations such as the National Institute for Health Research. ${ }^{7}$ It has been shown to improve research quality, efficiency of enrolment and participant satisfaction and well-being ${ }^{7-10}$ and may make trials accessible to a more diverse, therefore more representative, patient cohort.

Oncology leads the field in identifying ways to make research patient-centred. ${ }^{11-13}$ The findings provide insight into the significance and complexity of the discipline and have some transferability to patients across all disease groups. However, the trials landscape in one disease may be very different from another disease. Factors such as number of competing trials, disease characteristics, predominant demographics of the patient population and available treatments appear to influence participant engagement in research. ${ }^{14-18}$

Our team analysed data arising from the 'Patient Experiences of Research Participation' study. ${ }^{19}$ Participants with chronic obstructive pulmonary disease and obstructive sleep apnoea underwent semi-structured interviews after completing a trial at our site. A key finding was that even when interviewing only patients with respiratory disease, individual disease characteristics and cohort demographics appeared to influence patients' interaction with research and their reasons for engaging in trials. ${ }^{19}$ This builds on our view that the factors influencing the decision to participate in trials cannot be reliably extrapolated from other disease groups and supported our decision to lead a CF specific exploration.

This exploration included literature review (Registered on PROSPERO, CRD42020184886), focus groups and a national Delphi study conducted with relevant stakeholders. These mixed method approaches built a rich picture of the breadth of factors that influence engagement in trials by people with $\mathrm{CF}$ and identified several potentially modifiable barriers/facilitators to participation. ${ }^{20}$ Many of these would be simple to implement and have already led to practice changes locally and beyond. Others require a fundamental overhaul of trial design and delivery. Following this work, we identified six key concepts within the CF trial landscape which we believe require a more robust understanding of how they are perceived by people with $\mathrm{CF}$ and how they affect their decisions whether or not to participate in a trial:

- Does the type of medicine being investigated influence people's willingness to participate?

- Are people with CF prepared to attend a site different from their usual clinical centre for trial visits, and how far are they prepared to travel?

- Does stipend provision influence the decision to take part in trials, and what level of stipend is optimal?

- Are drug washout periods acceptable to patients?

- Are placebo-controlled trials acceptable to patients?
- Does the mechanism of accessing the experimental medicine after the trial is finished influence the decision to participate, and what are the preferred methods of access?

We plan to use a discrete choice experiment (DCE) to quantify the effect of these key features on decisions to take part in trials. By gaining a quantitative understanding of how these factors interact and influence patients' decisions to participate in trials, we hope to gain insight into these more controversial aspects of trial design and delivery. The European Clinical Trials Network (CTN) protocol review committee reviews all submissions and provides feedback to sponsors, which is mandatory for any trials being run through the network. CTN is supportive of our work and, as appropriate, will adjust their processes in light of our findings. The Clinical Trials Accelerator Platform (CTAP) is a UK based CF trials network, which has similarly agreed to incorporate our findings into their review processes. This means that our findings can realistically influence trial delivery across Europe and beyond to optimise the acceptability of clinical trials to people with CF.

\section{AIMS}

\section{Primary}

- To elicit and quantify preferences of people with $\mathrm{CF}$ for clinical trials' features.

- To estimate the relative importance of these features when choosing to participate in a clinical trial.

\section{Secondary/exploratory}

- To explore differences in preferences across subgroups of respondents (such as perceived disease severity and access to modulator therapy through clinic).

\section{METHODS}

\section{Methodological choice}

Classical experimental methods investigating decision making are constrained by practical and ethical difficulties. Descriptive methods introduce numerous sources of bias and necessitate small sample sizes, posing questions about validity and generalisability. DCEs bridge this gap. They are based on the principle that products or services comprise multiple features, and that an individual's choice of a product or service is a function of the utility of each feature. ${ }^{21}$ Thus, DCEs can be used to elicit preferences for features of a product or a service. Statistical testing of pooled results from a sample of participants allows researchers to investigate the relative value that participants place on multiple features when making complex decisions. DCEs have been used to elicit health preferences since before 1990 in a wide range of healthcare areas. ${ }^{21} 22$

Our six questions can be isolated and defined as a feature, or attribute, with two or more fixed levels. We will use the DCE to quantify which attributes and levels exert 
Table 1 Showing the defined attributes and levels to be investigated in the discrete choice experiment

\begin{tabular}{|c|c|}
\hline $\begin{array}{l}\text { Technical } \\
\text { attribute }\end{array}$ & Levels \\
\hline $\begin{array}{l}\text { Type of } \\
\text { medicine }\end{array}$ & $\begin{array}{l}\text { Modulators } \\
\text { Mucolytics and anti-inflammatories } \\
\text { New antibiotics }\end{array}$ \\
\hline $\begin{array}{l}\text { Where you } \\
\text { attend your } \\
\text { trial visits }\end{array}$ & $\begin{array}{l}\text { Your usual clinical centre } \\
\text { Any CF centre within } 1.5 \text { hours of your home } \\
\text { Any CF centre within } 3 \text { hours of your home } \\
\text { Any CF centre in the UK which is more than } \\
3 \text { hours away from your home }\end{array}$ \\
\hline $\begin{array}{l}\text { The stipend } \\
\text { you may } \\
\text { receive }\end{array}$ & $\begin{array}{l}\text { No additional stipend } \\
£ 10 \text { stipend per visit } \\
£ 30 \text { stipend per visit } \\
£ 70 \text { stipend per visit }\end{array}$ \\
\hline $\begin{array}{l}\text { What } \\
\text { happens with } \\
\text { your usual } \\
\text { medicines }\end{array}$ & $\begin{array}{l}\text { No washout period } \\
\text { 4-week washout of modulator } \\
\text { 4-week washout of non-modulator }\end{array}$ \\
\hline Trial design & $\begin{array}{l}\text { Placebo controlled } \\
\text { Open label }\end{array}$ \\
\hline $\begin{array}{l}\text { Access to } \\
\text { the medicine } \\
\text { after the trial }\end{array}$ & $\begin{array}{l}\text { No special access } \\
\text { Priority access to later trials } \\
\text { Open label extension phase } \\
\text { Managed access programme }\end{array}$ \\
\hline
\end{tabular}

CF, cystic fibrosis.

the strongest influence on people with $\mathrm{CF}$ when deciding to accept/decline participation.

\section{Study design}

The first step of designing the DCE is defining attributes and levels that answer the research questions. Identifying the most appropriate attributes and levels is critical to ensuring the study's validity and relevance. ${ }^{23}{ }^{24}$ The attributes and levels should be representative of the range of scenarios that participants may encounter and sufficiently specific and distinguishable to minimise interactive effects. The team used mixed methods preliminary work and their extensive experience in CF trials to establish attributes and levels that mimic realistic scenarios and choices in the current or future trials landscape (table 1). The number of attributes ${ }^{6}$ and levels $^{2-4}$ represent a balanced set of options and are consistent with several high quality DCEs published in healthcare. ${ }^{22}$

These attributes and levels are then combined to create hypothetical choice tasks for participants (figure 1). As participants are never obliged to take part in clinical trials, the choice participants are offered is 'trial A' or 'trial B' or 'no trial' rather than requiring a forced response to participate.

\begin{tabular}{|c|c|c|c|}
\hline Trial Feature & Trial A & Trial B & No Trial \\
\hline The Type of medicine & $\begin{array}{c}\text { Mucolytics and } \\
\text { anti-inflammatories }\end{array}$ & Modulators & \multirow{6}{*}{$\begin{array}{l}\text { I would not take } \\
\text { part in either of } \\
\text { these trials }\end{array}$} \\
\hline $\begin{array}{l}\text { Where you will attend } \\
\text { your trial visits }\end{array}$ & $\begin{array}{l}\text { Any CF centre } \\
\text { within } 3 \text { hours of } \\
\text { your home }\end{array}$ & $\begin{array}{c}\text { Any CF centre in the } \\
\text { Uk which is more than } \\
3 \text { hours away from } \\
\text { your home }\end{array}$ & \\
\hline $\begin{array}{l}\text { The stipend (payment) } \\
\text { you may receive }\end{array}$ & $\begin{array}{l}\text { f30 stipend per } \\
\text { visit }\end{array}$ & No additional stipend & \\
\hline $\begin{array}{l}\text { What happens with } \\
\text { your usual medicines }\end{array}$ & $\begin{array}{c}\text { You would stop } \\
\text { your usual } \\
\text { modulator } \\
\text { medicine } 4 \text { weeks } \\
\text { before day } 1 \text { of the } \\
\text { trial }\end{array}$ & $\begin{array}{l}\text { You would stop one of } \\
\text { your usual (non- } \\
\text { modulator) medicines } \\
4 \text { weeks before day } 1 \\
\text { of the trial }\end{array}$ & \\
\hline Trial design & Open-label & Placebo-controlled & \\
\hline $\begin{array}{l}\text { Access to the } \\
\text { medicine after the } \\
\text { trial }\end{array}$ & $\begin{array}{c}\text { Open-label } \\
\text { extension phase }\end{array}$ & $\begin{array}{c}\text { Priority access to later } \\
\text { trials }\end{array}$ & \\
\hline
\end{tabular}

\begin{tabular}{|lccc|}
\hline $\begin{array}{l}\text { Which trial would you } \\
\text { take part in? }\end{array}$ & Trial A & Trial B & No Trial \\
\hline
\end{tabular}

Figure 1 Figure showing the layout of an example discrete choice experiment choice to be presented to participants.

Once levels and attributes were defined, we developed a survey instrument. The instrument contains a standard introduction, an explanation to assist participants in understanding the attributes they are being asked to consider and an outline of the task. It describes the fixed features of the trial that remain constant such as the visit schedule and length of study, modified from three current large-scale CF trials. The text is punctuated by quiz questions to keep participants engaged and confirm their understanding of the complex concepts in the study. The instrument also includes screening questions to determine eligibility and questions to collect baseline demographic characteristics and other details such as location of clinical centre and access to modulator therapy.

$\mathrm{RD}$ and $\mathrm{MB}$ completed pretests with people with $\mathrm{CF}$, purposively sampled to represent a range of trials experience. Subjects were asked to read the background information and compete the quiz questions to identify areas where the task was unclear or the concepts difficult to follow. Changes were incorporated after each test. The pretest instrument contained a set of choice tasks. We asked subjects to talk through their decisions so we could check that they could clearly identify the options and make a trade-off when considering each choice set. This allowed the study team to identify previously unnoticed confounders and refine the attributes and levels. Pretests 5 and 6 did not reveal any new concerns so pre-testing was ended after six participants.

The combination of levels used to define each profile, the set of profiles in each choice question, and the full set of choice questions in a DCE is known as the experimental design. These combinations of profiles must have statistical properties that allow estimation of the main-effect preference weights of interest. A full factorial design can be produced, in which every possible combination of level and attribute is presented to the participants. However, for many DCEs, this would result in hundreds of choice tasks. Considering the attributes and levels in our survey, 
a full factorial design would generate 1152 combinations for each alternative $\left(4^{3} \times 3^{2} \times 2\right)$, resulting in a design that includes 1327104 questions. This would be impossible for participants to complete with any consideration- resulting in meaningless data. A fractional factorial experimental design overcomes this challenge by presenting participants with fewer choice tasks with specific combinations of attributes and levels.

The minimum number of tasks that can generate a wellbalanced optimal design with six attributes with 4, 3 and 2 levels to be presented to a sample of 200 respondents is 72. The pilot testing found our target audience were able to engage well with 12 choice tasks. Thus, our final experimental design was based on presenting 12 choice tasks. Therefore, the experimental design was split into 6 blocks, comprising 12 choice tasks each. We used the commonly employed D-optimal algorithm with no prior assumptions as to how respondents would answer to construct a fractional factorial experimental design. ${ }^{25} 26$

The final survey instrument was programmed into the internet-based data collection software, Qualtrics. Each participant will be randomised to receive one of the six blocks. Within each block, the questions will be randomly presented in different orders to minimise ordering effects and the impact of potential learning and fatigue. The survey will be administered online to allow participation across the whole of the UK. Qualtrics can be programmed to include a user-friendly interface with useful features including the 'tool tip' function, whereby participants can hover over the attributes and levels and receive a reminder description of what the options mean.

\section{Sample size}

Sample size calculations are challenging in DCEs because the models used to estimate preference weights simultaneously estimate multiple coefficients, and researchers often have little prior expectations of effect sizes. Most published choice experiments have sample sizes between 100 and 300 respondents. ${ }^{27}$ However, minimum sample size depends on various criteria, including the complexity of the choice task and the desired precision of the results. ${ }^{27}$ Considering the results from the pretest, the attributes and levels included, and the modelling approach planned, we assume a sample of 200 respondents will be sufficient to achieve the study's aims.

\section{Recruitment}

We will advertise the survey over multiple social media platforms including Twitter, Facebook and Instagram. CTAP will advertise the survey in their newsletter. We will aim for a sample of 200 respondents, with no maximum sample size and no formal stratification. We will close the survey when we have recruited 200 people or at 8 weeks, whichever happens latest. If we over-recruit in 8 weeks, we will use the data from all participants.

\section{Inclusion criteria}

Standards of care and access to clinical trials vary dramatically across the globe. ${ }^{28}$ Therefore, the relative effect of trial features in the UK on the decision to take part in trials may be very different to another country. To maximise the utility of this study, we are only opening the survey to those receiving care in the UK. Currently, we only have the resources to conduct the survey online and in English.

- Person with CF.

- Age 16+.

- Receives their clinical care within the UK.

- Able to read English.

- Willing to consent to participation.

Participants will be asked to self-confirm eligibility based on three screening questions; age, location of clinical care and whether they have CF. Those who do not meet the eligibility will be unable to proceed with the survey.

\section{Withdrawal}

Participants are free to stop the survey at any time without giving reasons. The opening participant information explains their right to do so, and a statement that this will not impact on their right to access clinical care or clinical trials. An email address for the team will be provided in case participants have questions. No identifiable data will be collected, so if patients choose to withdraw, no follow-up will be attempted and reasons for withdrawal cannot be sought.

\section{Patient public involvement}

Patient engagement is critical to ensure the success and relevance of this study. The key themes to explore, as well as the relevant attributes and levels were identified through a series of interviews, focus groups and a Delphi survey, thus more than 90 patients and 30 healthcare professionals working within the field of CF contributed to the study's development. The draft survey was pretested and adjusted following feedback from six people with $\mathrm{CF}$ with a range of trial participation experience. We will disseminate the results in patient accessible forums to ensure the findings reach people with CF.

\section{Study timelines}

Survey development ran from November 2019 to June 2020. Survey administration and data collection will be conducted from October to December 2020. Analysis will be conducted from January to April 2021.

\section{ANALYSIS}

\section{General considerations}

Descriptive statistics will be used to record respondent characteristics and questions not in the DCE. The crosssectional DCE data will be explored using conditional multinomial logit model. ${ }^{29}$ We will use mixed logit models, such as random-parameters logit (RPL) and 
latent class (LC) models, ${ }^{30} 31$ to analyse the choice-format data. Unobserved variation in preferences across the sample can bias estimates in conventional conditionallogit choice models. The RPL and the LC models avoid this bias by estimating a distribution of preferences around each model parameter that accounts for variations among individual preferences. ${ }^{30} 3233$ In an attempt to explore the drivers of preference heterogeneity, RPL subgroup and LC analysis with the membership probability function modelled including respondents' characteristics will be implemented to better explore preference variation across the sample by identifying heterogeneity. ${ }^{34}$

Analyses will be performed in STATA 16.

\section{Description of respondent characteristics}

Descriptive analyses will be provided for each question not included in the DCE. For continuous and ordinal variables, number of available observations, mean, SD, median, minimum, IQR and maximum will be calculated. For categorical variables, number of available observations and frequency and percentage in each response category will be reported. Analyses will be performed on observed data only, and no imputation for missing values will be performed. The number of missing responses will be displayed for each question; denominators will not include missing values.

\section{Preference analysis}

\section{Utility specification}

The number of choice questions in the experimental design is expected to be sufficient to estimate main effects. Main effects include the preference weight for each attribute level independent of the other attributes and levels included in the study (table 1), using effects-coding for each categorical attribute level. A linear and continuous function will be tested and compared with a categorical representation for the stipend attribute, implementing the best to fit the data in the final model.

\section{Determination of the appropriate model estimation approach}

Each respondent will answer 12 questions from the experimental design, each of which offers the participant to select one of two trials, or to say that they would not participate in either trial. The DCE questions generate cross-sectional data that require analysis using advanced statistical techniques. ${ }^{35}$ An RPL model will be used to analyse the DCE data collected and estimate the primary endpoints (ie, all attribute-level preference weights) assuming all random parameters are normally distributed and independent unless goodness-of-fit measures indicate that an RPL model assuming all random parameters are normally distributed and correlated better fits the data. An LC model will also be used to analyse the DCE data, comparing it with the final specification of the RPL model. Goodness of fit, as well as the optimal number of classes in the LC model, will be evaluated using the Bayesian information criterion ${ }^{36}$ and the Akaike information criterion. ${ }^{38}$

\section{Conditional relative attribute importance}

To determine the relative importance of an attribute, conditional relative importance will be calculated as the difference between the attribute level with the highest preference weight and the level with the lowest preference weight. This difference represents the maximum change in conjoint utility achievable with any attribute, given the levels chosen for the attributes in the study. The conditional relative importance of an attribute also describes the relative importance of each attribute relative to all other attributes included in the study, conditional on the range of levels of the attribute.

\section{Analysis of preference heterogeneity}

Subgroup analysis will be used to determine whether average preferences vary among respondents in subgroups for selected attributes (eg, perceived disease severity and access to modulators through clinic). In addition to exploring preference heterogeneity using a set of predefined subgroups in the RPL analysis, the LC model can also be used to explore preference heterogeneity by including covariates in the membership probability function, based on the same subgroups of interest. ${ }^{31} 34$

\section{Incomplete data}

Respondents may choose not to answer some questions in the DCE. All respondents who answer at least one DCE choice question will be included in the analysis and descriptive summary statistics. Respondents who do not answer any choice questions will be excluded from the analysis and descriptive summary statistics. Respondents who choose not to answer certain demographic or treatment history questions will be noted in the summary statistics, and their responses will be included in the DCE. If a question is missed, the survey will be programmed to give a reminder to participants to input a value.

\section{STUDY VALIDITY}

Discrete-choice experiments have been widely employed in recent decades to quantify preferences for new and existent treatments and devices in a variety of clinical applications and settings ${ }^{22}$ and is considered by the authors the best approach to address our research question among multiple stated preference approaches. ${ }^{22}$ The design was developed following good research practices as defined by International Society for Pharmacoeconomics and Outcomes Research guidelines and standard practice in preference studies. ${ }^{39}$ The DCE has a number of strengths derived from the use of best practices. ${ }^{23}$ The survey was built on extensive preliminary investigation, significant experience of the study team and close collaboration with invested stakeholders. It was pretested with a well-balanced and representative population. The trial-choice data will be analysed using RPL methods following good research practices $^{35}$ that avoid estimation bias from unobserved 
variation in preferences across the sample and withinsample correlation in the choice sequence for each respondent.

\section{Internal validity}

\section{Measurement error/misclassification}

Measurement error increases with task difficulty, which decreases the precision with which coefficients (ie, preference weights) can be estimated for any given sample size. Therefore, the number of attributes included in the final survey instrument has been limited to those that are necessary to answer the research questions.

The data collected are based on responses to hypothetical design or implementation features selected for a hypothetical clinical trial. These choices are intended to simulate possible enrolment decisions but do not have the same consequences of actual, real-world enrolment and may not have the same impact. Thus, differences can arise between stated and actual choices. We have attempted to limit potential hypothetical bias by constructing choice questions that mimic realistic design choice features as closely as possible and that map clearly into the current trials landscape.

\section{Information bias}

Information necessary to take the survey will be provided to all respondents. Participants may be unfamiliar with some of the concepts, therefore the explanations risk presenting information bias. The study team have collaborated with people with $\mathrm{CF}$ to refine the language and reduce biased presentation of potential attributes and levels.

\section{Selection bias}

Selection bias is a potential limitation of this study, and all voluntary online surveys. Research has shown that results from online stated-preference studies are, in general, not statistically different from those elicited through faceto-face interviews. ${ }^{40}{ }^{41} \mathrm{We}$ will use multiple social media forums to attempt to reach out to a maximally diverse cohort. Finally, the preferences of those who agree to participate in this study may be systematically different from preferences of those who do not agree to participate. The team will be unable to measure or control for this bias.

\section{Residual confounding}

Patient choice could potentially be confounded with unobserved variables, including prior experience or unobserved sociodemographic characteristics. In addition, because this study is limited to eliciting preferences for a subset of potential outcomes and features of trials, patients may make assumptions about outcomes and features that are not included when completing the DCE questions. We will try to mitigate the latter effect through survey pretesting; however, these remain potential study limitations.

\section{External validity of study design}

Although there are standards and good practices for stated-preference studies, ${ }^{23}$ no definitive guidance exists to ensure a preference study is externally valid.

\section{ETHICS SUMMARY}

The principal investigator (JCD) has obtained approval from the Joint Research Compliance Office at Imperial College London. The questionnaire opens with an embedded participant information page. Consent to enter the study will be sought from participants following this full explanation through a question asking patients to confirm their willingness to participate. The right of the participant to refuse to participate without giving reasons will be respected and stated explicitly in the consent information. Participants are free to withdraw at any time and this will be made clear in the opening statements. A participant will be considered to have withdrawn if they do not complete the questionnaire. If the respondent withdraws from the study no further evaluations will be performed, and no additional data will be collected. The study team may retain and continue to use any data collected before such withdrawal of consent. No identifying information will be collected. Therefore, confidentiality will be maintained throughout this study.

\section{DISSEMINATION POLICY}

On study completion, the data will be analysed and tabulated and a final study report prepared. The report will form the basis of peer-reviewed publications. The findings will be presented at international conferences and patient accessible forums. A summary sheet will be generated for participants and circulated via the same channels used to advertise the survey.

\section{Author affiliations}

${ }^{1}$ National Heart Lung institute, Imperial College London, London, UK

${ }^{2}$ Department of Paediatric Respiratory Medicine, Royal Brompton Hospital, London, UK

${ }^{3}$ Health Preference Assessments, RTI Health Solutions, Belfast, UK

${ }^{4}$ School of Medicine, Dentistry and Biomedical Sciences, Queens University of Belfast, Belfast, UK

${ }^{5}$ UKCRC Centre of Excellence for Public Health (NI), Queen's University Belfast, Belfast, UK

${ }^{6}$ Department of Adult Cystic Fibrosis, Royal Brompton Hospital, London, UK

Acknowledgements With thanks to all of the people with CF who were involved in this study, from developing themes and pretesting the survey instrument, as well as those who take part in our survey.

Contributors RAD and MB cowrote the protocol, with RAD's focus on refining the research question, attributes and levels and practical and regulatory aspects of study design and administration and MB's focus primarily on experimental design and analysis. SE, FK and SM provided their expert input and experience to enhance the protocol at all stages. JCD is the principal investigator and provided ultimate oversight for the protocol design and development.

Funding The authors have not declared a specific grant for this research from any funding agency in the public, commercial or not-for-profit sectors.

Competing interests $\mathrm{RD}, \mathrm{MB}, \mathrm{FK}$ and SM have no competing interests to declare. JCD has served on advisory boards and participated in clinical trial leadership, 
educational activities and grant review board activities for a number of pharma companies active in CF clinical trials: Vertex, PTI, Galapagos, AbbVie, AlgiPharma, Chiesi, Enterprise, Teva, Ionis, Eloxx, Roche and Gilead. JSE has provided consultancy and advice for Vertex, Celtaxsys, Corbus, Ionis in clinical trial design and delivery. He also holds an EU Innovative medicines Initiative grant with Novartis, Polyphor and Alaxia.

Patient and public involvement Patients and/or the public were involved in the design, or conduct, or reporting, or dissemination plans of this research. Refer to the Methods section for further details.

\section{Patient consent for publication Not required.}

Provenance and peer review Not commissioned; externally peer reviewed.

Open access This is an open access article distributed in accordance with the Creative Commons Attribution Non Commercial (CC BY-NC 4.0) license, which permits others to distribute, remix, adapt, build upon this work non-commercially, and license their derivative works on different terms, provided the original work is properly cited, appropriate credit is given, any changes made indicated, and the use is non-commercial. See: http://creativecommons.org/licenses/by-nc/4.0/.

\section{ORCID iD}

Rebecca Anne Dobra http://orcid.org/0000-0002-6857-0988

\section{REFERENCES}

1 Fibrosis Foundation C. Patient registry annual data report, 2018.

2 Heijerman HGM, McKone EF, Downey DG, et al. Efficacy and safety of the elexacaftor plus tezacaftor plus ivacaftor combination regimen in people with cystic fibrosis homozygous for the F508del mutation: a double-blind, randomised, phase 3 trial. Lancet 2019;394:1940-8.

3 Middleton PG, Mall MA, Dřevínek P, et al. Elexacaftor-tezacaftorivacaftor for cystic fibrosis with a single Phe508del allele. $N$ Engl $J$ Med 2019;381:1809-19.

4 Mayer-Hamblett N, Boyle M, VanDevanter D. Advancing clinical development pathways for new CFTR modulators in cystic fibrosis. Thorax 2016;71:454-61.

5 Dekkers JF, Berkers G, Kruisselbrink E, et al. Characterizing responses to CFTR-modulating drugs using rectal organoids derived from subjects with cystic fibrosis. Sci Trans/ Med 2016;8:344ra84.

6 Clinical Trials Finder. CFF clinical trials tool [online]. Available: https:// www.cff.org/Trials/Finder [Accessed 29 Aug 2020].

7 NIHR. Involve patients [online]. Available: https://www.nihr.ac.uk/ health-and-care-professionals/engagement-and-participation-inresearch/involve-patients.htm [Accessed 29 Aug 2020].

8 Briel M, Elger B, von Elm E, et al. Insufficient recruitment and premature discontinuation of clinical trials in Switzerland: qualitative study with trialists and other stakeholders. Swiss Med Wkly 2017;147:w14556.

9 Sacristán JA, Aguarón A, Avendaño-Solá C, et al. Patient involvement in clinical research: why, when, and how. Patient Prefer Adherence 2016;10:631-40.

10 et alDomecq JP, Prutsky G, Elraiyah T. Patient engagement in research: a systematic review [online], 2014. Available: http://www. biomedcentral.com/1472-6963/14/89 [Accessed 26 Jun 2019].

11 Bell JAH, Balneaves LG. Cancer patient decision making related to clinical trial participation: an integrative review with implications for patients' relational autonomy. Support Care Cancer 2015;23:1169-96.

12 Horn L, Keedy VL, Campbell N, et al. Identifying barriers associated with enrollment of patients with lung cancer into clinical trials. Clin Lung Cancer 2013;14:14-18.

13 Walsh E, Sheridan A. Factors affecting patient participation in clinical trials in Ireland: a narrative review. Contemp Clin Trials Commun 2016;3:23-31.

14 Unger JM, Hershman DL, Albain KS, et al. Patient income level and cancer clinical trial participation. J Clin Oncol 2013;31:536-42.

15 Kasner SE, Del Giudice A, Rosenberg S, et al. Who will participate in acute stroke trials? Neurology 2009;72:1682-8.

16 Del Giudice A, Plaum J, Maloney E, et al. Who will consent to emergency treatment trials for subarachnoid hemorrhage? Acad Emerg Med 2009;16:309-15.

17 Goode PS, Fitzgerald MP, Richter HE, et al. Enhancing participation of older women in surgical trials. J Am Coll Surg 2008;207:303-11.
18 Behrendt CE, Hurria A, Tumyan L, et al. Socioeconomic and clinical factors are key to uncovering disparity in accrual onto therapeutic trials for breast cancer. J Natl Compr Canc Netw 2014;12:1579-85.

19 Dobra R, Guilmant E, Higgins T. Understanding and improving participants' experience of health research; Patient evaluation of research participation in a dedicated respiratory biomedical reserach unit (BRU) Clinical resreach facility (CRF). In: Thorax, British Thoracic Society Winter Meeting, The Queen Elizabeth II Centre Broad Sanctuary Westminster London SW1P 3EE 2 to 4 December 2015 Programme and Abstracts [online] 2017;70 http://thorax.bmj.com/ content/70/Suppl_3.toc

20 Dobra R, Elborn S, Madge S, et al. P361 what influences participation in clinical trials by people with cystic fibrosis? A national Delphi study. J Cystic Fibrosis 2020;19:S157.

21 Clark MD, Determann D, Petrou S, et al. Discrete choice experiments in health economics: a review of the literature. Pharmacoeconomics 2014;32:883-902.

22 Soekhai V, de Bekker-Grob EW, Ellis AR, et al. Discrete choice experiments in health economics: past, present and future. Pharmacoeconomics 2019;37:201-26.

23 Bridges JFP, Hauber AB, Marshall D, et al. Conjoint analysis applications in health-a checklist: a report of the ISPOR good research practices for conjoint analysis Task force. Value Health 2011;14:403-13.

24 Hollin IL, Craig BM, Coast J, et al. Reporting formative qualitative research to support the development of quantitative preference study protocols and corresponding survey instruments: guidelines for authors and reviewers. Patient 2020;13:121-36.

25 Kuhfeld WF, Tobias RD, Garratt M. Efficient experimental design with marketing research applications. J Mark Res 1994;31:545-57.

26 Techniques G, Kuhfeld WF. Marketing research methods in SAS, 2010.

27 Marshall D, Bridges JFP, Hauber B, et al. Conjoint analysis applications in health-how are studies being designed and reported?: An update on current practice in the published literature between 2005 and 2008. Patient 2010;3:249-56.

28 Bell SC, Mall MA, Gutierrez H, et al. The future of cystic fibrosis care: a global perspective. Lancet Respir Med 2020;8:65-124.

29 McFadden D. Conditional logit analysis of qualitative choice behavior. Front Econom 1974.

30 McFadden D, Train K. Mixed MNL models for discrete response. $J$ Appl Econ 2000;15:447-70.

31 Greene WH, Hensher DA, Greene W. A latent class model for discrete choice analysis: contrasts with mixed logit. Trans Res Part B: Methodol 2003;37:681-98.

32 Louviere JJ, Flynn TN, Carson RT. Discrete choice experiments are not conjoint analysis. J Choice Model 2010;3:57-72.

33 Train K, Sonnier G. Mixed logit with bounded distributions of correlated partworths, 2004.

34 Boeri M, Saure D, Schacht A, et al. Modeling heterogeneity in patients' preferences for psoriasis treatments in a multicountry study: a comparison between Random-Parameters Logit and latent class approaches. Pharmacoeconomics 2020;38:593-606.

35 Hauber AB, González JM, Groothuis-Oudshoorn CGM, et al. Statistical methods for the analysis of discrete choice experiments: a report of the ISPOR conjoint analysis good research practices Task force. Value Health 2016;19:300-15.

36 Kanarek NF, Tsai H-L, Metzger-Gaud S, et al. Geographic proximity and racial disparities in cancer clinical trial participation. $J$ Natl Compr Canc Netw 2010;8:1343-51.

37 Hurvich CM, Tsai CL. Regression and time series model selection in small samples. Biometrika 1989;76:297-307.

38 McLachlan G, Peel D. Finite mixture models [online]. Hoboken, NJ: John Wiley \& Sons, Inc, 2000. http://doi.wiley.com/10.1002/ 0471721182

39 Reed Johnson F, Lancsar E, Marshall D, et al. Constructing experimental designs for discrete-choice experiments: report of the ISPOR conjoint analysis experimental design good research practices Task force. Value Health 2013;16:3-13.

40 Nielsen. How social media impacts brand marketing [online]. Available: https://www.nielsen.com/us/en/insights/article/2011/howsocial-media-impacts-brand-marketing/ [Accessed 3 Sep 2020].

41 Marta-Pedroso C, Freitas H, Domingos T. Testing for the survey mode effect on contingent valuation data quality: a case study of web based versus in-person interviews. Ecological Economics 2007;62:388-98. 